ARTIGO ORIGINAL

\title{
Correlação entre percepção subjetiva do esforço e fadiga neuromuscular dos membros inferiores em atletas de futebol
}

\section{The correlation between the rating of perceived exertion and neuromuscular fatigue on the lower body in soccer athletes}

Caique Lopes Müller ${ }^{1}$, Pedro Sotero da Cunha Neto ${ }^{1}$, Eloir Junior da Silva dos Santos ${ }^{1}$, Alexandre Oliveira ${ }^{1}$, Rosimeide Francisco dos Santos Legnani ${ }^{2}$, Elto Legnani ${ }^{1}$

${ }^{1}$ Universidade Tecnológica Federal do Paraná (UTFPR), Curitiba/PR, Brasil

${ }^{2}$ Universidade Estadual de Ponta Grossa (UEPG), Ponta Grossa/PR, Brasil

\section{HISTÓRICO DO ARTIGO}

Recebido: 06 junho 2020

Revisado: 16 julho 2019

Aprovado: 22 julho 2020

\section{PALAVRAS-CHAVE:}

Futebol; Esporte; Fadiga Muscular.

\section{KEYWORDS:}

Soccer; Sports; Muscle Fatigue.

\section{RESUMO}

OBJETIVO: Determinar a correlação entre a percepção subjetiva do esforço (PSE) e a fadiga neuromuscular dos membros inferiores por meio de saltos mensurados no pré e pós treino em atletas de futebol.

MÉTODOS: Quatorze atletas de futebol (sub 17) foram monitorados ao longo de quatro sessões de treinamento. Com as seguintes características: estatura $(173 \pm 6,81 \mathrm{~cm})$, peso $(64,43 \pm 5,80 \mathrm{~kg})$ e percentual de gordura $(14,74 \pm 3,11)$. A PSE foi monitorada por meio do aplicativo e-trimp, 15 minutos após o final de cada sessão de treinamento. Os saltos foram avaliados por meio da plataforma de salto Jump System Pro, 10 minutos, antes e depois das sessões de treinamento. Os dados foram analisados no programa estatístico SPSS por meio da estatística descritiva e correlação de Spearman.

RESULTADOS: A correlação entre a PSE e a diferença da potência relativa do salto vertical (pré/pós treino) foi fraca para todas as sessões. As sessões 1 e 4 apresentaram correlação negativa $(r=-0,184, p=0,530 ; r=-0,159$, $p=0,587)$. As sessões 2 e 3 apresentaram correlação positiva $(r=0,010, p=0,973 ; r=0,373, p=0,188)$, respectivamente.

CONCLUSÃO: Não foram observadas correlações estatisticamente significativas entre a PSE e a potência relativa de salto nos atletas avaliados.

\section{ABSTRACT}

OBJECTIVE: Define the correlation between the rating of perceived exertion (RPE) and the power of the lower limbs before and after training in soccer athletes.

METHODS: Were monitored 14 soccer athletes (under 17 years old) over four training sessions. With the following characteristics: height $(173 \pm 6.81 \mathrm{~cm})$, weight $(64.43 \pm 5.80 \mathrm{~kg})$ and fat percentage $(14.74 \pm 3.11)$. RPE was monitored through the e-trimp app, 15 minutes after the end of each training session. Jumps were evaluated through the Jump System Pro jumping platform, 10 minutes before and after the training sessions. Data was analyzed in the SPSS statistical program using descriptive statistics and Spearman correlation.

RESULTS: The correlation between the RPE and the difference of the relative power of the vertical jump test (pre/post training) was weak for all the sessions. Sessions 1 and 4 presented a negative correlation $(r=-0.184, p=0.530 ; r=-0.159, p=0.587)$. Sessions 2 and 3 showed a positive correlation $(r=0.010, p=0.973$; $r=0.373, p=0.188)$, respectively.

CONCLUSION: There were no statistically significant correlations between the RPE and the relative jumping power in the evaluated athletes. 


\section{INTRODUÇÃO}

Para jogar futebol profissionalmente, faz-se necessário vários requisitos, dentre eles: preparação física, técnica, tática e psicológica (CORRÊA et al., 2002). Nesse sentido, um planejamento ideal quanto a preparação física de jogadores de futebol, deve reunir os indicadores de frequência, volume, intensidade, bem como, o controle das cargas de treinamento das sessões (CASAMICHANA et al., 2013; HADER et al., 2019; SILVA, 2017; THORPE et al., 2017).

Durante os treinamentos ou nas partidas oficiais de futebol, é possível observar a queda no desempenho dos jogadores, que pode ser explicada pela fadiga muscular que ocorre devido ao estresse oxidativo ou funcional, o que limita o desempenho físico, técnico e tático dos atletas, aumentando o risco de lesões esportivas (INTERNATIONAL OLYMPIC COMMITTEE INJURY AND ILLNESS EPIDEMIOLOGY CONSENSUS GROUP, 2020). Nesse particular, com os objetivos de aumentar o rendimento esportivo, com maior segurança e menor risco de lesões nos atletas, os preparadores físicos e fisiologistas vêm utilizando diversos métodos de controle de carga durante as sessões de treinamento (BRANCACCIO; MAFFULLI; LIMONGELLI, 2007; FOSTER et al., 2001; NAKAMURA; MOREIRA; AOKI, 2010; HADER et al., 2019; SILVA, 2017).

Uma das estratégias de controle de cargas de treinamento que vem ganhando popularidade no meio esportivo é o da percepção subjetiva de esforço (PSE), principalmente pela sua simplicidade e baixo custo de utilização (COUTTS et al., 2009; DAL PUPO et al., 2010; CASAMICHANA et al., 2013; IMPELLIZIERI et al., 2013; NAKAMURA; SALDANHA; AOKI, 2011). Esse método tem sido testado em atletas de natação (BARROSO et al., 2011), de judô (VIVEIROS et al., 2014), futebol profissional masculino (BORIN et al., 2011), feminino (ALEXIOU; COUTTS, 2008) e entre atletas jovens (KUNRATH et al., 2016; GONÇALVES et al., 2016; 2016 NAIDU et al., 2019).

Alguns pesquisadores tem dedicado esforços em verificar a relação entre a carga interna perceptual e indicadores neuromo-tores relacionados à potência dos membros inferiores (DAL PUPO, 2010; KUNRATH et al., 2016; GONÇALVES e tal., 2016), desta-cando a potencialização do rendimento dos atletas, por meio do controle de carga e otimização da recuperação muscular. Neste particular, avaliações utilizando-se de protocolos que incluíram saltos (ROSCHEL et al. 2011), seja ao monitoramento da perfor-mance (ABAD et al., 2016; FLÁVIO; DE OLIVEIRA; DE SOUZA, 2018);) ou para avaliar a fadiga aguda em resposta ao treinamento (KUNRATH et al., 2016; HADER et al., 2019; BUCHHEIT et al., 2017; THORPE et al., 2017) ou, ainda, ao longo prazo (BORIN et al., 2011). Entretanto, estudos com atletas jovens ainda são escassos (KUNRATH et al., 2016), logo, como forma de contribuir o corpo de conhecimento científico dessa área, o presente estudo objetiva-se em avaliar a relação entre percepção subjetiva de esforço e a fadiga da potência de membros inferiores via salto vertical (SV).

\section{MÉTODOS}

Para o estudo participaram 14 atletas de futebol do sexo masculino, todos nascidos no ano de 2002 (17 anos), com estatura média de $173 \pm 6,81 \mathrm{~cm}$, peso médio de $64,43 \pm 5,80 \mathrm{~kg}$ e percentual de gordura média de $14,72 \pm 3,11$. A presente pesquisa apresentou um delineamento descritivo correlacional, examinando-se a relação entre determinadas variáveis. Todos os atletas eram praticantes de futebol de campo, com pelo menos dois anos de experiência, treinavam cinco vezes na semana, com sessões de 90 minutos de duração e pertenciam a uma categoria de base de um clube de futebol da cidade de Curitiba/ $\mathrm{PR}$, com relação a fase de treinamentos, encontravam-se no meio da temporada do campeonato da Federação do Estado na categoria Sub17.

Todos foram autorizados pelos pais ou responsáveis, mediante a assinatura de Termo de Consentimento Livre e Esclarecido (TCLE), bem como do Termo de Assentimento Livre e Esclarecido (TALE), concordando em participar da pesquisa. A participação foi voluntária e todos os cuidados para garantir o anonimato e o sigilo das informações dos atletas foram atendidos. O projeto foi submetido para avaliação no Comitê de Ética em Pesquisa do Setor de Ciências da Saúde da Universidade Federal Tecnológica do Paraná, sendo aprovado de acordo com a Resolução Nº. 453/2012 do Conselho Nacional de Saúde, sob Parecer $\mathrm{N}^{\circ}$. 2.812.040 e registro número 92184318.2.0000.5547 (CAD).

A coleta de dados dos atletas foi realizada no local dos treinamentos, durante quatro sessões de treinamento (90 minutos/sessão), no mês de outubro de 2018. Os treinamentos tiveram as seguintes características: primeiro dia (Sessão 1): no pri-meiro dia, foi realizado um trabalho resistido em forma de circuito, seguido por jogos reduzidos (JR) de 8 jogadores contra 4. Segundo dia (Sessão 2) foi realizado exercícios de mobilidade e core, seguido do trabalho com bola realizado com 10 jogadores contra 5+1 coringa sem gols e depois com gols e goleiros. No terceiro dia (Sessão 3) foi realizado um aquecimento, para então iniciar-se com um trabalho de transição 3 jogadores contra $2+1$ coringa, seguido por uma parte técnica específica por posição e terminando com uma liberação miofascial. No quarto dia (Sessão 4) foi realizado um JR de 8 jogadores contra 6 finalizando com o treinamento resistido em circuito.

Para a avaliação antropométrica foram mensuradas a massa corporal (com os atletas vestindo somente calção) e a estatura (com os atletas descalços), sendo aferidas, respectivamente, com uma balança da marca (Plena), precisão de 0,1 kg e uma fita métrica na parede com anteparo sobre a cabeça, no qual foram adotados os pontos extremos do corpo, calcâneo até o ponto mais alto da cabeça, desconsiderando o cabelo (GUEDES; GUEDES, 1998). Além disso, os atletas foram caracterizados quanto ao seu percentual de gordura, utilizando-se da equação de Slaughter et al. (1988). Portanto, foi avaliada a espessura das seguintes dobras cutâneas: subescapular e tríceps braquial, sendo utilizado para tal avaliação, o Plicômetro Científico Tradicional (Cescorf $\left.{ }^{\circledR}\right)$, com sensibilidade: $0,1 \mathrm{~mm}$ e pressão das molas $\left( \pm 10 \mathrm{~g} / \mathrm{mm}^{2}\right)$.

Para a coleta de dados da PSE dos atletas, foram adotados os seguintes procedimentos e equipamentos: a) cadastro da modalidade futebol, b) da equipe, c) dos atletas, d) das sessões de treinamento no aplicativo da web E-TRIMP' ${ }^{1}$. Todos os atletas já estavam bem ambientados sobre a utilização da escala de

\footnotetext{
Aplicação web que pode ser acessada em qualquer navegador através do seguinte link: http:// www.e-trimp.com br e também do aplicativo mobile desenvolvido para a plataforma Android e que pode ser encontrado na Play Store, no seguinte link: https:/ play. $\mathrm{d}=$ br.com.etrimp\&hl=pt BR.
} 
CR10 de Borg et al. (1982) e modificada por Foster et al. (2001), como experiência de pelo menos um ano, e, mesmo assim tinham acesso à visualização da escala no aplicativo. Estes relataram a PSE após 15 minutos das sessões de treinamento. Para facilitar o registro dos valores da PSE, um aparelho de celular foi disposto sobre uma mesa apropriada para ampará-lo, no qual o atleta estava sozinho para impedir que houvesse qualquer influência externa.

Em relação à potência de membros inferiores, a mesma foi avaliada por meio do teste de salto vertical (SV) com contra movimento e sem o auxílio das mãos. Para a realização do teste foi solicitado que o avaliado estivesse com as mãos fixas ao quadril e os joelhos totalmente estendidos. Ao iniciar o movimento o avaliado realizava uma rápida flexão do joelho até aproximadamente $90^{\circ}$, e em seguida realizava a rápida extensão dos joeIhos, buscando a maior altura do salto. $O$ teste foi feito pelos atletas cerca de 10 minutos antes e depois da sessão de treinamentos, na qual cada jogador saltou três vezes, respeitando um intervalo de 30 segundos, sendo registrado somente o melhor salto válido, recordando as variáveis(altura do salto em $\mathrm{cm}$, potência absoluta e potência relativa).

Foi realizado um aquecimento antes do salto pré-treino, na qual era composto por 5 minutos de trote no ritmo escolhido pelo atleta, mobilidade dinâmica, exercícios calistênicos e 5 saltos de familiarização. Os saltos foram realizados em cima da plataforma de salto Jump System Pro, sendo os dados traduzidos e tabulados automaticamente no Software Jump System 1.0 instalado em um notebook que foi ligado a plataforma. A coleta de dados foi realizada pelo pesquisador juntamente com o preparador físico da equipe.

Inicialmente, foi realizada a análise descritiva dos dados, posteriormente o teste de normalidade de Shapiro-Wilk foi utilizado para verificar a distribuição dos dados. Para determinar o grau de correlação entre a PSE e o SV, utilizou-se o teste de Spearman. Para verificar a diferença entre as potências relativas pré e pós, foi utilizado o teste dos sinais de Wilcoxon. Todas as análises foram realizadas no programa estatístico SPSS (versão 20 para Windows; SPSS, Inc. Chicago, IL), o nível de significância utilizado foi de $5 \%$, os gráficos foram organizados por meio do programa Microsoft Excel ${ }^{\circledR} 2013$.

\section{RESULTADOS}

As médias das diferenças entre a altura de salto (pré pós sessão), foram positivas para às três primeiras sessões $(0,50 \pm 2,41 ; 0,71 \pm 3,68 ; 0,86 \pm 3,20)$. Diferenças negativas foram encontradas nos resultados da quarta sessão de treino em relação à altura de salto $(-0,21 \pm 1,47)$ e potência absoluta $(-3,93$ $\mathrm{cm})$, consequentemente, a maior média de PSE foi observada na sessão 4 (Tabela 1).

A Tabela 2 apresenta os valores de correlação entre a PSE, diferença de potência absoluta e diferença de potência relativa das alturas de salto para as quatro sessões de treinamento avaliadas. As correlações variaram de $r=0,02(p \leq 0,93)$ a $r=-0,46(p \leq 0,09)$ para a potência absoluta e de $r=-0,18(p \leq 0,53)$ a $r=-0,15(p \leq 0,58)$ para a potência relativa, no entanto, não apresentaram significância estatística.

Tabela 3 apresenta os valores médios e o desvio padrão, assim como os valores de significância com relação a diferença entre os valores observados no SV pré e pós sessão de treino. Não foram observadas diferenças estatísticas entre os valores analisados.

Tabela 1. Média das diferenças entre a potência absoluta (pré- pós sessão de treino) e percepção subjetiva de esforço de atletas de futebol da categoria sub $16(n=14)$.

\begin{tabular}{|c|c|c|c|c|}
\hline Sessões & Mínimo & Máximo & Média & $\begin{array}{l}\text { Desvio } \\
\text { Padrão }\end{array}$ \\
\hline \multicolumn{5}{|c|}{ \# altura do SV pré e pós sessão de treino $(\mathrm{cm})$} \\
\hline 1'a sessão & -5 & 5 & 0,86 & 3,20 \\
\hline 2a sessão & -3 & 5 & 0,50 & 2,41 \\
\hline 3ạ sessão & -7 & 10 & 0,71 & 3,68 \\
\hline 4ạ sessão & -3 & 1 & $-0,21$ & 1,47 \\
\hline \multicolumn{5}{|c|}{ f potência absoluta pré e pós sessão de treino (watts) } \\
\hline 1a sessão & -87 & 121 & 30,14 & 71,23 \\
\hline 2ạ sessão & -57 & 123 & 25,21 & 51,25 \\
\hline 3ạ sessão & -246 & 301 & 24,57 & 114,04 \\
\hline 4ạ sessão & -84 & 57 & $-3,93$ & 41,51 \\
\hline \multicolumn{5}{|c|}{ Percepção subjetiva de esforço das sessões } \\
\hline 1 a & 4 & 8 & 6,07 & 0,99 \\
\hline $2^{\mathrm{a}}$ & 6 & 10 & 7,29 & 1,06 \\
\hline 3 a & 5 & 8 & 6,21 & 0,80 \\
\hline $4 \underline{a}$ & 5 & 9 & 7,64 & 1,08 \\
\hline
\end{tabular}

$\overline{\mathrm{SV}}=$ Salto Vertical; $\neq=$ valor da altura de salto pré treino menos a altura de salto pós treino

Tabela 2. Correlação entre a PSE e a diferença entre a potência absoluta (PWA) e relativa (PWR), pré e pós sessão de treino $(n=14)$.

\begin{tabular}{|c|c|c|}
\hline PWA & $r$ & $p$ \\
\hline PSE $1 x \neq$ PWA 1 & 0,02 & 0,93 \\
\hline PSE $2 x \neq$ PWA 2 & 0,16 & 0,57 \\
\hline PSE $3 x \neq$ PWA 3 & 0,29 & 0,31 \\
\hline PSE $4 x \neq$ PWA 4 & $-0,46$ & 0,09 \\
\hline \multicolumn{3}{|l|}{ PWR } \\
\hline PSE $1 x \neq$ PWR 1 & $-0,18$ & 0,53 \\
\hline PSE $2 x \neq$ PWR 2 & 0,01 & 0,97 \\
\hline PSE $3 x \neq$ PWR 3 & 0,37 & 0,18 \\
\hline PSE $4 x \neq$ PWR 4 & $-0,15$ & 0,58 \\
\hline
\end{tabular}

Tabela 3. Diferença entre a potência relativa do SV (pré- pós sessão de treino) em atletas de futebol sub-16 $(n=14 ; p<0,05)$

\begin{tabular}{lccc}
\hline PWR pré e pós $(\mathbf{W} / \mathbf{k g})$ & Média & Desvio Padrão & $\mathbf{p}$ \\
\hline PWR pré 1 & 26,14 & 2,24 & 0.47 \\
PWR pós 1 & 25,93 & 2,05 & \\
PWR pré 2 & 26,36 & 1,73 & 0.23 \\
PWR pós 2 & 26,07 & 1,59 & \\
PWR pré 3 & 26,07 & 1,97 & 0.94 \\
PWR pós 3 & 26,00 & 2,03 & \\
PWR pré 4 & 26,29 & 1,81 & 0.25 \\
PWR pós 4 & 26,50 & 1,95 & \\
\hline
\end{tabular}

$\mathrm{SV}=$ Salto Vertical; $\mathrm{PWR}=$ Potência relativa da sessão; $\mathrm{W} / \mathrm{kg}=$ Watts por $\mathrm{kg}$

Os resultados demonstraram que a diferenças entre a altura SV pré e pós sessão de treino foram positivas e menores que 1 centímetro para as três primeiras sessões de treinos analisadas, 
sendo negativa para a quarta sessão de treino $(-0,21 \pm 1,47 \mathrm{~cm})$. Da mesma forma, as correlações entre PSE e potência absoluta e relativa, foram positivas para as três primeiras sessões de treino $(0,50 \pm 2,41 ; 0,71 \pm 3,68 ; 0,86 \pm 3,20)$ e negativa para a quarta sessão de treino $(-0,21 \pm 1,47)$.

As correlações observadas foram de fraca a moderada magnitude para todas as variáveis analisadas e nenhuma delas apresentou significância estatística. Ao analisar os resultados das correlações, levando em consideração os tipos de treinamento aplicados aos atletas, percebe-se que todas as sessões contemplaram componentes técnicos e táticos. Entretanto, a primeira e a quarta sessão de treinamento, incluíram exercícios resistidos como um dos componentes das sessões. O acréscimo dos exercícios resistidos pode ter impactado na fadiga dos atletas na primeira e na quarta sessões de treino, isso pode ter refletido na maior média da PSE observadas nessas sessões de treino e nas maiores diferenças entre os saltos pré e pós sessão, observadas na primeira e quarta sessão de treino respectivamente, consequentemente impactando nos indicadores de correlação negativa observados.

Estudos com atletas profissionais de futebol indicam que a PSE é um método válido e confiável ao controle das cargas de trei-namento (FOSTER et al., 2001; IMPELLIZZERI et al., 2004; ALEXIOU; COUTTS, 2008; NAKAMURA et al., 2010), no entanto, quando recorre-se a literatura sobre SV como forma de controle de carga, percebe-se uma inconsistência quanto aos resultados encontrados. Nesse sentido, os resultados encontrados vão ao encontro do estudo realizado por Kunrath et al. (2016), que indicaram não haver diferença estatisticamente significativas entre os valores do SV, pré e pós treino (técnico e tático) e, em treinamento de jogo reduzido, seguido de treino de sprints repetidos com mudança de direção em jovens futebolistas (SILVA, 2017).

A relação entre carga interna e externa, encontra-se resultados mais consistentes e promissores entre atletas adultos, tanto no futebol (FOSTER et al., 2001; IMPELLIZZERI et al., 2004; ALEXIOU; COUTTS, 2008), quanto em outras modalidades (BARROSO et al., 2014; NAKAMURA et al., 2010). Dessa forma, esperava-se encontrar nos atletas desse estudo uma queda no desempenho no SV em todas as sessões de treinamento. A correlação negativa de moderada intensidade, entre a PSE das sessões de treinamento com o SV e a potência (Absoluta e relativa) encontradas na quarta sessão de treino, demonstrou uma leve tendência de impacto da carga de treino na fadiga dos atletas. Esse resultado é promissor e sinaliza à necessidade de se realizar mais estudos com atletas de categorias de base, haja vista a importância de melhor compreender o impacto das cargas de treino no organismo de atletas jovens.

Da mesma forma, é importante destacar que o desempenho em testes neuromotores, podem sofrer diversos tipos de influências negativas por parte dos atletas, tais como: a baixa motivação dos atletas no momento do salto e a biomecânica do movimento, esses fatores podem ter afetado o desempenho dos atletas na altura do SV. Isso pode justificar a necessidade de se realizar novos estudos, com amostras maiores e com atletas de diferentes categorias, sobretudo, com maiores cuidados na ela-boração das sessões de treinamento e na execução dos testes.

O presente estudo apresenta limitações relacionadas à pequena quantidade de atletas e sessões analisadas, a não avalia- ção do status de maturação dos mesmos, a falta de instrumentos de controle de carga interna mais consistentes, como por exemplo, a frequência cardíaca, além disso, a falta de controle de fatores ligados ao estado motivacional dos atletas pode ter interferido nos resultados dos testes de SV. Para futuros estudos, sugere-se correlacionar a PSE com outras ferramentas de controle de intensidade como frequência cardíaca, avaliar o status de maturação e utilizar outras variáveis do SV como o tempo de contração muscular no SV e tempo de voo no SV, buscando variáveis mais sensíveis para avaliar a fadiga dos atletas. Aspectos como, a realização da coleta de dados em ambiente real de treinamento, a utilização de atletas de categorias de base, o uso de equipamento para avaliar o SV de boa resolução, são fatores positivos e relevantes do estudo.

\section{CONCLUSÃO}

Não foram encontradas correlações estatisticamente significativas entre a percepção subjetiva do esforço e a potência dos membros inferiores pré e pós treino nos atletas analisados. Entretanto, os resultados encontrados na análise da primeira e quarta sessão de treinamento, indicaram uma discreta correlação negativa entre a PSE, altura de salto e potência absoluta e relativa nos atletas analisados.

\section{REFERÊNCIAS}

ABAD, C.; CUNIYOCHI, R.; KOBAL, R.; GIL, S.; PASCOTO, K.; NAKAMURA, F. LOTURCO, I. Efeito do destreinamento na composição corporal e nas capacidades de salto vertical e velocidade de jovens jogadores da elite do futebol brasileiro. Revista Andaluza de Medicina del Deporte, Sevilla, v. 9, n. 3, p. 124-30, 2016.

ALEXIOU, H.; COUTTS, A. J. A comparison of methods used for quantifying internal training load in women soccer players. International Journal of Sports Physiology and Performance, Champaign, v. 3, n. 3, p. 320-30, 2008.

VIVEIROS, L.; COSTA, E. C.; MOREIRA, A.; NAKAMURA, F. Y.; AOKI, M. S. Monitoramento do treinamento no judô: comparação entre a intensidade da carga planejada pelo técnico e a intensidade percebida pelo atleta. Revista Brasileira de Medicina do Esporte, São Paulo, v. 17, n. 4, p. 266-9, 2011.

BARROSO, R.; CARDOSO, R. K.; CARMO, E. C.; TRICOLI, V. Perceived exertion in coaches and youngs wimmers with different training experience. International Journal of Sports Physiology and Performance, Champaign, v. 9, n. 2, p. 212-6, 2014

BORG, G. A. V. Psychophysical bases of perceived exertion. Medicine and Science in Sports and Exercise, Indianapolis, v. 14, n. 5, p. 377-81, 1982.

BORIN, J. P.; OLIVEIRA, R. S. D.; CAMPOS, M. G. D.; CREATTO, C. R.; PADOVANI, C. R. P.; PADOVANI, C. R. Evaluación del efecto del entrenamiento em el período preparatório en atletas profesionales del fútbol. Revista Brasileira de Ciências do Esporte, Brasília, v. 33, n. 1, p. 219-33, 2011.

BRANCACCIO, P.; MAFFULLI, N.; LIMONGELLI, F. M. Creatine kinase monitoring in sport medicine. British Medical Bulletin, Oxford, v. 81, n. 1, p. 20930, 2007.

BUCHHEIT, M.; LACOME, M.; CHOLLEY, Y.; SIMPSON, B. M. Neuromuscular responses to conditioned soccer sessions assessed via GPS-embedded accelerometers: insights into tactical periodization. International Journal of Sports Physiology and Performance, Champaign, v. 13, n. 5, p. 577-83, 2018

CASAMICHANA, D.; CASTELLANO, J.; CALLEJA-GONZALEZ, J.; SAN ROMÁN, J. CASTAGNA, C. Relationship between indicators of training load in soccer players. The Journal of Strength \& Conditioning Research, Colorado Springs, v. 27, n. 2, p. 369-74, 2013.

CORRÊA, D. K. D. A.; ALCHIERI, J. C.; DUARTE, L. R. S.; STREY, M. N. Excelência na produtividade: a performance dos jogadores de futebol profissional. Psicologia: Eeflexão e Crítica, Porto Alegre, v. 15, n. 2, p. 447-60, 2002. 
COUTTS, A. J.; RAMPININI, E.; MARCORA, S. M.; CASTAGNA, C.; IMPELLIZZERI, F. M. Heart rate and blood lactate correlates of perceived exertion during small-sided soccer games. Journal of Science and Medicine in Sport, Belconnen, v. 12, n. 1, 79-84, 2009.

DAL PUPO, J.; ALMEIDA, C. M. P.; DETANICO, D.; SILVA, J. F. D.; GUGLIELMO, L. G. A.; SANTOS, S. G. D. Potência muscular e capacidade de sprints repetidos em jogadores de futebol. Revista Brasileira de Cineantropometria \& Desempenho Humano, Florianópolis, v. 12, n. 4, p. 255-61, 2010.

FLÁVIO, J. M.; DE OLIVEIRA, D. C. X.; DE SOUZA, E. G. Efeito do treinamento pliométrico no desempenho da velocidade e altura dos saltos vertical e horizontal para jovens jogadores de futebol. Revista Brasileira de Futsal e Futebol, São Paulo, v. 10, n. 41, p. 673-80, 2018.

FOSTER, C.; FLORHAUG, J. A.; FRANKLIN, J.; GOTTSCHALL, L.; HROVATIN, L. A.; PARKER, S.; DODGE, C. A new approach to monitoring exercise training. The Journal of Strength \& Conditioning Research, Colorado Springs, v. 15, n. 1, p. 109-15, 2001

GONÇALVES, L. G. C.; AQUINO, R. L. D. Q. T.; PUGGINA, E. F. Caracterização do perfil de jovens jogadores de futebol: uma análise das habilidades técnicas e variáveis antropométricas. Motricidade, Ribeira de Pena, v. 12, n. 2 , p. 27-37, 2016

GUEDES, D. P.; GUEDES, J. E. R. P. Controle do peso corporal: composição corporal, atividade física e nutrição. Londrina: Midiograf. 311 p.

HADER, K.; RUMPF, M. C.; HERTZOG, M.; KILDUFF, L. P.; GIRARD, O.; SILVA, J. R. Monitoring the athlete match response: Can external load variables predict post-match acute and residual fatigue in soccer? A systematic review with meta-analysis. Sports Medicine-Open, Auckland, v. 5, n. 1, p. 48, 2019.

IMPELLIZZERI, F. M.; RAMPININI, E.; COUTTS, A. J.; SASSI, A. L. D. O.; MARCORA, S. M. Use of RPE-based training load in soccer. Medicine \& Science in Sports \& Exercise, Indianapolis, v. 36, n. 6, p. 1042-7, 2004.

INTERNATIONAL OLYMPIC COMMITTEE INJURY AND ILLNESS EPIDEMIOLOGY CONSENSUS GROUP. International Olympic Committee Consensus Statement: methods for recording and reporting of epidemiological data on injury and illness in sports 2020 (Including the STROBE Extension for Sports Injury and IIIness Surveillance (STROBE-SIIS). Orthopaedic Journal of Sports Medicine, New York, v. 8, n. 2, p. 2325967120902908, 2020.

KUNRATH, C. A.; GONÇALVES, E.; SILVA, L. F. D. S.; TIGGEMANN, C. L.; DIAS, C. P.; OLIVEIRA, U. O. D.; TEOLDO, I. Avaliação da intensidade do treinamento técnico-tático e da fadiga causada em jogadores de futebol da categoria sub-20. Revista Brasileira de Educação Física e Esporte, São Paulo, v. 30, n. 2, p. $217-25,2016$

NAIDU, S. A.; FANCHINI, M.; COX, A.; SMEATON, J.; HOPKINS, W. G.; SERPIELLO, F. R. Validity of session rating of perceived exertion assessed via the cr100 scale to track internal load in elite youth football players. International Journal of Sports Physiology Performance, Hanover, v. 14, n. 3, p. 403-6, 2019.

NAKAMURA, F. Y.; MOREIRA, A.; AOKI, M. S. Monitoramento da carga de treinamento: a percepção subjetiva do esforço da sessão é um método confiável. Journal of Physical Education, Maringá, v. 21, n. 1, p. 1-11, 2010.

ROSCHEL, H.; TRICOLI, V.; UGRINOWITSCH, C. Treinamento físico: considerações práticas e científicas. Revista Brasileira de Educação Física e Esporte, São Paulo, v. 25, n. Especial, p. 53-65, 2011.

SLAUGHTER, M. H.; LOHMAN, T. G.; BOILEAU, R.; HORSWILL, C. A.; STILLMAN, R. J.; VAN LOAN, M. D.; BEMBEN, D. A. Skinfold equations for estimation of body fatness in children and youth. Human Biology, Nova York, v. 60 , n. 5 , p. $709-23,1988$

SILVA, M. E. S. da. Resposta afetiva nos métodos de treinamento do futebol em jovens: uma comparação entre jogo reduzido e treino de sprints repetidos com mudança de direção. 2017. 15f. TCC (Graduação em Educação Física) - Universidade Federal do Rio Grande do Norte, Natal, 2017.

THORPE, R. T.; STRUDWICK, A. J.; BUCHHEIT, M.; ATKINSON, G.; DRUST, B.; GREGSON, $W$. The influence of changes in acute training load on daily sensitivity of morning-measured fatigue variables in elite soccer players. International Journal of Sports Physiology and Performance, Champaign, v. 12 , n. s2, p. S2-107, 2017.

\section{CONFLITO DE INTERESSE}

Os autores do estudo declaram não haver conflito de interesses.

\section{ORCID E E-MAIL DOS AUTORES}

Caique Lopes Müller

ORCID: 0000-0002-1034-7847.

E-mail: caiquemuller@hotmail.com

Pedro Sotero da Cunha Neto (Autor Correspondente)

ORCID: 0000-0002-8771-0181.

E-mail: soteroneto@gmail.com

Eloir Junior da Silva dos Santos

ORCID: 0000-0001-5127-0359.

E-mail: profjuniorcap@gmail.com

Alexandre Oliveira

ORCID: 0000-0002-4786-2623.

E-mail: alexoli2690@hotmail.com

Rosimeide Francisco dos Santos Legnani

ORCID: 0000-0001-7604-3056.

E-mail: legnanirosi@gmail.com

Elto Legnan

ORCID: 0000-0002-8251-8000.

E-mail: legnanielto@gmail.com 